\begin{tabular}{|c|c|c|}
\hline $\begin{array}{r}\text { PRAMANA } \\
- \text { journal of } \\
\text { physics }\end{array}$ & (c) Indian Academy of Sciences & $\begin{array}{l}\text { Vol. } 76 \text {, No. } 4 \\
\text { April 2011 } \\
\text { pp. 533-542 }\end{array}$ \\
\hline
\end{tabular}

\title{
Travelling wave solutions to nonlinear physical models by means of the first integral method
}

\author{
İSMAIIL ASLAN \\ Department of Mathematics, Izmir Institute of Technology, Urla, Izmir 35430, Turkey \\ E-mail: ismailaslan@iyte.edu.tr
}

MS received 21 April 2010; revised 8 October 2010; accepted 9 November 2010

\begin{abstract}
This paper presents the first integral method to carry out the integration of nonlinear partial differential equations in terms of travelling wave solutions. For illustration, three important equations of mathematical physics are analytically investigated. Through the established first integrals, exact solutions are successfully constructed for the equations considered.
\end{abstract}

Keywords. First integral method; modified Boussinesq equation; Fornberg-Whitham equation; nonlinear Schrödinger equation; travelling wave solutions.

PACS Nos 02.30.Jr; 02.30.Hq; 04.20.Jb

\section{Introduction}

Many modelling problems arising in nonlinear physical sciences (such as plasma physics, crystal lattice theory, fluid mechanics, etc.) deal with nonlinear partial differential equations (NPDEs) with rich structures. The investigation of exact and explicit solutions to NPDEs is an important and attractive research area. Not all NPDEs are integrable. For the last four decades or so, many methods have been successfuly developed by a diverse group of scientists to carry out the integration of NPDEs in terms of analytic solutions. Backlund transformation method [1], Painleve expansion method [2], inverse scattering method [3], symmetry method [4], Hirota's bilinear method [5], sine-cosine method [6], tanh-coth method [7], Jacobi elliptic function method [8], $\left(G^{\prime} / G\right)$-expansion method [9], exp-function method [10] are to name a few. However, most of the existing methods have certain disadvantages and they require tedious and complicated algebraic calculations.

On the other hand, considerable attention has been given to problem of finding special types of analytic solutions to understand biological, physical and chemical phenomena modelled by NPDEs. Among the possible solutions, certain solutions may depend only on a single combination of variables such as travelling wave variables. Recently, applying the theory of commutative algebra, Feng [11-13] proposed a new approach which is currently called the first integral method (or the algebraic curve method) to study NPDEs from an 


\section{Ismail Aslan}

analytical point of view. The first integral method has proven to be useful for constructing travelling wave solutions to a variety of nonlinear problems (see, for example [14-21] and the references therein). The method is reliable, effective, precise and does not require complicated and tedious computations. The main idea of the first integral method is to find first integrals of nonlinear differential equations in polynomial form. Taking the polynomials with unknown polynomial coefficients into account, the method provides exact and explicit solutions. But the application of the first integral method to NPDEs having distinct physical properties is still an interesting and important problem.

In this paper, we wish to show the applicability of the first integral method to some physically important NPDEs such as the modified Boussinesq equation, the Fornberg-Whitham equation, the (2+1)-dimensional nonlinear Schrödinger (NLS) equation, and present some improved results. The paper is organized as follows: In $\$ 2$, we summarize the method to make the paper self-contained. In $\S 3$, the problems are analysed and in $\S 4$, a brief conclusion is provided.

\section{The first integral method}

In this section, we initiate our study by briefly reviewing the procedure. Let us consider a nonlinear partial differential equation for a function $u$ of two real variables, space $x$ and time $t$ :

$$
P\left(u, u_{t}, u_{x}, u_{t t}, u_{t x}, u_{x x}, \ldots\right)=0,
$$

where $P$ is a polynomial in its arguments and subscripts denote partial derivatives. By means of the transformation $u(x, t)=U(\xi), \xi=k x+w t+\xi_{0}$, where $k, w$ and $\xi_{0}$ are arbitrary constants, we reduce eq. (1) to an ordinary differential equation (ODE) of the form

$$
P\left(U, k U^{\prime}, w U^{\prime} k^{2} U^{\prime \prime}, k w U^{\prime \prime}, w^{2} U^{\prime \prime}, \ldots\right)=0,
$$

where $U=U(\xi)$ and the primes denote ordinary derivatives with respect to $\xi$. Next, we introduce a new independent variable

$$
V(\xi)=U(\xi), \quad W(\xi)=U^{\prime}(\xi),
$$

which leads to a system of ODEs of the form

$$
\begin{aligned}
V^{\prime}(\xi) & =W(\xi), \\
W^{\prime}(\xi) & =Q(V(\xi), W(\xi)) .
\end{aligned}
$$

In general, it is a difficult and challenging task to solve planar systems of ODEs, such as eq. (4), directly. Thus, based on the qualitative theory of ordinary differential equations [22], if we can find a single first integral to the two-dimensional autonomous system (4), we can reduce eq. (2) to a first-order ODE which may be easier to solve. But, there is neither a systematic theory that can tell us how to find the first integrals of eq. (4), nor is there a logical way for telling us what these first integrals are. At this point, our key idea will be to use the division theorem for two variables in the complex domain $\mathbb{C}$ to find a first integral to eq. (4): 
Division theorem. Suppose that $P(w, z)$ and $Q(w, z)$ are polynomials in $\mathbb{C}[w, z]$ and $P(w, z)$ is irreducible in $\mathbb{C}[w, z]$. If $Q(w, z)$ vanishes at all zero points of $P(w, z)$, then there exists a polynomial $G(w, z)$ in $\mathbb{C}[w, z]$ such that $Q(w, z)=P(w, z) G(w, z)$.

The division theorem can be proved by using complex theory of several variables $[11,23]$ or by the following Hilbert-Nullstellensatz theorem from the ring theory of commutative algebra [24]:

Hilbert-Nullstellensatz theorem. Let $k$ be a field and $L$ an algebraic closure of $k$. Then

(i) Every ideal $\gamma$ of $k\left[X_{1}, \ldots, X_{n}\right]$ not containing 1 admits at least one zero in $L^{n}$.

(ii) Let $\mathbf{x}=\left(x_{1}, \ldots, x_{n}\right)$ and $\mathbf{y}=\left(y_{1}, \ldots, y_{n}\right)$ be two elements of $L^{n}$. For the set of polynomials of $k\left[X_{1}, \ldots, X_{n}\right]$ zero at $\mathbf{x}$ to be identical with the set of polynomials of $k\left[X_{1}, \ldots, X_{n}\right]$ zero at $\mathbf{y}$, it is necessary and sufficient that there exists a $k$-automorphism sof $L$ such that $y_{i}=s\left(x_{i}\right)$ for $1 \leq i \leq n$.

(iii) For an ideal $\alpha$ of $k\left[X_{1}, \ldots, X_{n}\right]$ to be maximal, it is necessary and sufficient that there exists an $\mathbf{x}$ in $L^{n}$ such that $\alpha$ is the set of $k\left[X_{1}, \ldots, X_{n}\right]$ zero at $\mathbf{x}$.

(iv) For a polynomial $Q$ of $k\left[X_{1}, \ldots, X_{n}\right]$ to be zero on the set of zeros in $L^{n}$ of an ideal $\gamma$ of $k\left[X_{1}, \ldots, X_{n}\right]$, it is necessary and sufficient that there exists an integer $m>0$ such that $Q^{m} \in \gamma$.

Remark 1. It is well-known that the real field $\mathbb{R}$ is a subfield of the complex field $\mathbb{C}$. Hence, we can always extend a given equation in $\mathbb{R}$ to an equation in $\mathbb{C}$. If the extended equation has an algebraic curve solution in $\mathbb{C}$, then the intersection of the manifold of this solution and the real plane must be the algebraic curve solution of the original equation in $\mathbb{R}$. This means that the Division Theorem stated in $\mathbb{C}$ can also be used in $\mathbb{R}$ [11].

\section{Applications}

In this section, we investigate three NPDEs using the first integral method for the first time.

\subsection{The modified Boussinesq equation}

Let us consider the modified Boussinesq equation [25] which reads

$$
u_{t t}=u_{x x}+\left(u^{3}\right)_{x x}+u_{x x x x},
$$

where $u=u(x, t)$. Equation (5) can be regarded as the continuous limit of a Fermi Pasta Ulam dynamical system with cubic nonlinearity. Yan et al [26] have obtained some similarity reductions of eq. (5). Now, to look for travelling wave solutions of eq. (5), we first make the transformation $u(x, t)=U(\xi), \xi=x-c t+\xi_{0}$, where $c$ and $\xi_{0}$ are arbitrary constants. Then, integrating the resultant equation twice and setting the integration constants to zero for simplicity, we get

$$
U^{\prime \prime}=\left(c^{2}-1\right) U-U^{3},
$$




\section{Ismail Aslan}

where the prime denotes derivative with respect to $\xi$ and $U=U(\xi)$. Let $z=U$ and $y=U^{\prime}$. Then eq. (6) can be rewritten as the two-dimensional autonomous system

$$
\left\{\begin{array}{l}
\frac{\mathrm{d} z}{\mathrm{~d} \xi}=y, \\
\frac{\mathrm{d} y}{\mathrm{~d} \xi}=\left(c^{2}-1\right) z-z^{3} .
\end{array}\right.
$$

Now, suppose that $z=z(\xi)$ and $y=y(\xi)$ are nontrivial solutions of eq. (7). Also, assume that $q(z, y)=\sum_{i=0}^{m} A_{i}(z) y^{i}$ is an irreducible polynomial in the complex domain $\mathbb{C}$ such that

$$
q(z(\xi), y(\xi))=\sum_{i=0}^{m} A_{i}(z) y^{i}=0,
$$

where the polynomials $A_{i}(z)(i=0,1, \ldots, m)$ are relatively prime in $\mathbb{C}$ with $A_{m}(z) \equiv 0$. Equation (8) is a first integral of eq. (7). We note that $\mathrm{d} q / \mathrm{d} \xi$ is a polynomial in $z$ and $y$. Thus, $q(z(\xi), y(\xi))=0$ implies that $\mathrm{d} q / \mathrm{d} \xi=0$. Then, by the Division Theorem, there exists a polynomial $B(z)+C(z) y$ in the complex domain $\mathbb{C}$ such that

$$
\frac{\mathrm{d} q}{\mathrm{~d} \xi}=\frac{\partial q}{\partial z} \frac{\mathrm{d} z}{\mathrm{~d} \xi}+\frac{\partial q}{\partial y} \frac{\mathrm{d} y}{\mathrm{~d} \xi}=(B(z)+C(z) y)\left[\sum_{i=0}^{m} A_{i}(z) y^{i}\right] .
$$

We consider the case $m=2$ of eq. (8). Hence, taking eqs (7) and (9) into account, we get

$$
\begin{aligned}
\sum_{i=0}^{2}\left[A_{i}^{\prime}(z) y^{i+1}\right]+\sum_{i=0}^{2}\left[i A_{i}(z) y^{i-1}\left(\left(c^{2}-1\right) z-z^{3}\right)\right] \\
=[B(z)+C(z) y]\left[\sum_{i=0}^{2} A_{i}(z) y^{i}\right] .
\end{aligned}
$$

Equating the coefficients of $y^{i}(0 \leq i \leq 3)$ in eq. (10) leads to the system

$$
\begin{aligned}
& y^{3}: A_{2}^{\prime}(z)=C(z) A_{2}(z), \\
& y^{2}: A_{1}^{\prime}(z)=C(z) A_{1}(z)+B(z) A_{2}(z), \\
& y^{1}: A_{0}^{\prime}(z)=C(z) A_{0}(z)+B(z) A_{1}(z)+\left[2 z^{3}-2\left(c^{2}-1\right) z\right] A_{2}(z), \\
& y^{0}: B(z) A_{0}(z)+\left[z^{3}-\left(c^{2}-1\right) z\right] A_{1}(z)=0 .
\end{aligned}
$$

From eq. (11), we obtain $A_{2}(z)=c_{0} \exp \left(\int C(z) \mathrm{d} z\right)$, where $c_{0}$ is an integration constant. Since $A_{2}(z)$ and $C(z)$ are polynomials, we deduce that $C(z)=0$ and $A_{2}(z)$ must be a constant. For simplicity, we can take $A_{2}(z)=1$. Then, eqs (12) and (13) reduce to the following equations:

$$
\begin{aligned}
& A_{1}^{\prime}(z)=B(z), \\
& A_{0}^{\prime}(z)=B(z) A_{1}(z)+2 z^{3}-2\left(c^{2}-1\right) z .
\end{aligned}
$$




\section{Travelling wave solutions to nonlinear physical models}

Balancing the degrees of $A_{0}(z), A_{1}(z)$ and $B(z)$, we can conclude that deg $B(z)=1$ and $\operatorname{deg} A_{1}(z)=2$. Supposing $B(z)=b_{1} z+b_{0}\left(b_{1} \neq 0\right)$ and $A_{1}(z)=a_{2} z^{2}+a_{1} z+a_{0}\left(a_{2} \neq 0\right)$ in eq. (15), we get $b_{1}=2 a_{2}$ and $b_{0}=a_{1}$. Thus, from eq. (16), we have

$$
A_{0}(z)=\frac{a_{2}^{2}+1}{2} z^{4}+a_{1} a_{2} z^{3}+\frac{a_{1}^{2}+2 a_{0} a_{2}-2\left(c^{2}-1\right)}{2} z^{2}+a_{0} a_{1} z+d,
$$

where $d$ denotes an integration constant. By substituting $A_{0}(z), A_{1}(z)$ and $B(z)$ into eq. (14) and equating the coefficients of $z^{i}(0 \leq i \leq 5)$ to zero, we obtain the following system of nonlinear algebraic equations:

$$
\begin{aligned}
& z^{5}: 4 a_{2}+2 a_{2}^{3}=0, \\
& z^{4}: 3 a_{1}+5 a_{1} a_{2}^{2}=0, \\
& z^{3}: 2 a_{0}+6 a_{2}-6 c^{2} a_{2}+4 a_{1}^{2} a_{2}+4 a_{0} a_{2}^{2}=0, \\
& z^{2}: 4 a_{1}-4 c^{2} a_{1}+a_{1}^{3}+6 a_{0} a_{1} a_{2}=0, \\
& z^{1}: 2 a_{0}-2 c^{2} a_{0}+2 a_{0} a_{1}^{2}+4 d a_{2}=0, \\
& z^{0}: 2 d a_{1}=0 .
\end{aligned}
$$

Solving the system (eqs (18)-(23)) simultaneously, we get the solution set

$$
\begin{aligned}
& d=-\frac{1}{2}\left(1-2 c^{2}+c^{4}\right), \\
& a_{0}= \pm i\left(\sqrt{2}-\sqrt{2} c^{2}\right), \quad a_{1}=0, \quad a_{2}= \pm i \sqrt{2}
\end{aligned}
$$

where $c$ remains arbitrary. Now, taking the solution set (24) into account, eq. (8) becomes

$$
\begin{aligned}
- & \left(\frac{1}{2}\left(1-2 c^{2}+c^{4}\right)-\frac{1}{2}\left(2\left(1-c^{2}\right)-2 \sqrt{2}\left(\sqrt{2}-\sqrt{2} c^{2}\right)\right) z^{2}+\frac{z^{4}}{2}\right) \\
& \pm\left(i\left(\sqrt{2}-\sqrt{2} c^{2}\right)+i \sqrt{2} z^{2}\right) y+y^{2}=0
\end{aligned}
$$

which is a first integral of eq. (7). Solving eq. (25), we get

$$
y=\mp \frac{1}{2} i\left(\sqrt{2}-\sqrt{2} c^{2}+\sqrt{2} z^{2}\right) .
$$

Finally, combining eq. (7) with eq. (26) and changing to the original variables, we obtain travelling wave solutions to eq. (5) as

$$
\begin{array}{ll}
u_{1}^{\mp}(x, t)=\mp i \sqrt{1-c^{2}} \tanh \left[\sqrt{\frac{1-c^{2}}{2}}\left(x-c t+\xi_{0}\right)\right], & |c|<1, \\
u_{2}^{\mp}(x, t)=\mp i \sqrt{c^{2}-1} \tan \left[\sqrt{\frac{c^{2}-1}{2}}\left(x-c t+\xi_{0}\right)\right], & |c|>1,
\end{array}
$$

where $\xi_{0}$ is an arbitrary constant. 


\section{İsmail Aslan}

\subsection{The Fornberg-Whitham equation}

Let us consider the nonlinear dispersive wave equation, also known as the FornbergWhitham equation, in the form

$$
u_{t}-u_{x x t}+u_{x}+u u_{x}=u u_{x x x}+3 u_{x} u_{x x} .
$$

Equation (29) has appeared in the study of qualitative behaviours of wave-breaking [27, 28]. A peaked solution of the form $u(x, t)=K \exp \left(-\frac{1}{2}\left|x-\frac{4}{3} t\right|\right)$, where $K$ is an arbitrary constant, to eq. (29) was obtained by Fornberg and Whitham [29]. By employing the bifurcation method to dynamical systems, Zhou and Tian [30,31] have constructed kinklike, antikink-like, peakons and periodic cusp wave solutions to eq. (29). Now, applying the transformation $u(x, t)=U(\xi), \xi=x-c t+\xi_{0}$ to eq. (29) and integrating the resultant equation once, we get

$$
U^{\prime \prime}(U-c)+(c-1) U-\frac{1}{2} U^{2}+\left(U^{\prime}\right)^{2}=k,
$$

where the primes denote derivative with respect to $\xi$ and $k$ is an integration constant. Letting $z=U$ and $y=U^{\prime}$ in eq. (30), we obtain the equivalent planar system

$$
\left\{\begin{array}{l}
\frac{\mathrm{d} z}{\mathrm{~d} \xi}=y, \\
\frac{\mathrm{d} y}{\mathrm{~d} \xi}=\frac{(1-c) z+(1 / 2) z^{2}-y^{2}+k}{z-c} .
\end{array}\right.
$$

Now, we make the transformation $\mathrm{d} \xi=(z-c) \mathrm{d} \eta$ in eq. (31) to avoid the singular line $z=c$ temporarily. Thus, system (31) becomes

$$
\left\{\begin{array}{l}
\frac{\mathrm{d} z}{\mathrm{~d} \eta}=(z-c) y, \\
\frac{\mathrm{d} y}{\mathrm{~d} \eta}=(1-c) z+\frac{1}{2} z^{2}-y^{2}+k .
\end{array}\right.
$$

The systems (31) and (32) have the same topological structures except for the straight line $z=c$. For the new system (32), $z=c$ is its invariant straight line solution. We consider the case $m=1$ of eq. (8). From now on, we shall omit some details because the procedure is the same. Then, by equating the coefficients of $y^{i}(0 \leq i \leq 2)$ on both sides of eq. (9), we have

$$
\begin{aligned}
& y^{2}:(z-c) A_{1}^{\prime}(z)=(1+C(z)) A_{1}(z), \\
& y^{1}:(z-c) A_{0}^{\prime}(z)=C(z) A_{0}(z)+B(z) A_{1}(z), \\
& y^{0}: B(z) A_{0}(z)=\left[\frac{1}{2} z^{2}+(1-c) z+k\right] A_{1}(z) .
\end{aligned}
$$

As $A_{1}(z)$ and $C(z)$ are polynomials, from eq. (33), we deduce that $C(z)=-1$ and $A_{1}(z)$ must be a constant. For simplicity, we can take $A_{1}(z)=1$. Then, eq. (34) indicates that $\operatorname{deg} B(z) \leq \operatorname{deg} A_{0}(z)$. Thus, from eq. (35), we conclude that $\operatorname{deg} B(z) \leq \operatorname{deg} A_{0}(z)=1$. Assuming $A_{0}(z)=a_{1} z+a_{0}\left(a_{1} \neq 0\right)$ and $B(z)=b_{1} z+b_{0}\left(b_{1} \neq 0\right)$ in eq. (34), we get $b_{1}=2 a_{1}$ and $b_{0}=a_{0}-c a_{1}$. Substituting $A_{0}(z)$ and $B(z)$ into eq. (35) and setting the 


\section{Travelling wave solutions to nonlinear physical models}

coefficients of $z^{i}(0 \leq i \leq 2)$ to be zero, we derive a system of nonlinear algebraic equations for $a_{0}, a_{1}, k$ and $c$. Solving the resultant system simultaneously, we get the solution set

$$
k=\frac{1}{18}\left(8-18 c+9 c^{2}\right), \quad a_{0}= \pm \frac{1}{6}(4-3 c), \quad a_{1}= \pm \frac{1}{2},
$$

where $c$ remains arbitrary. Using the condition (36) in (8), we obtain

$$
y=\mp \frac{1}{2} z \mp \frac{1}{6}(4-3 c) .
$$

Combining eq. (31) with eq. (37) and changing to the original variables, we find an exact peaked wave solution to eq. (29) as

$$
u^{ \pm}(x, t)=c-\frac{1}{3}\left(4-\mathrm{e}^{ \pm \frac{1}{2}\left(x-c t+\xi_{0}\right)}\right)
$$

where $c$ and $\xi_{0}$ remain arbitrary.

\subsection{The (2+1)-dimensional NLS equation}

Let us consider the $(2+1)$-dimensional NLS equation

$$
i u_{t}+u_{x x}+u_{y y}+\mu|u|^{2} u=0,
$$

where $\mu$ is a real constant, $u=u(x, y, t)$ is a complex-valued function, and subscripts represent partial derivatives. To solve eq. (39), we first make the transformation

$$
u=U(\xi) \exp (i \eta), \quad \xi=k(x+l y+\lambda t), \quad \eta=\alpha x+\beta y+\gamma t .
$$

The substitution of the transformation (eq. (40)) into eq. (39) yields the ODE

$$
k^{2}\left(1+l^{2}\right) U^{\prime \prime}(\xi)+\mu U^{3}(\xi)-\left(\gamma+\alpha^{2}+\beta^{2}\right) U(\xi)=0,
$$

where $\lambda=-2(\alpha+\beta l)$ and the prime denotes derivative with respect to $\xi$. Let $z=U$ and $y=U^{\prime}$ in eq. (41), then we obtain the equivalent planar system

$$
\left\{\begin{array}{l}
\frac{\mathrm{d} z}{\mathrm{~d} \xi}=y, \\
\frac{\mathrm{d} y}{\mathrm{~d} \xi}=\frac{\gamma+\alpha^{2}+\beta^{2}}{k^{2}\left(1+l^{2}\right)} z-\frac{\mu}{k^{2}\left(1+l^{2}\right)} z^{3} .
\end{array}\right.
$$

We consider the case $m=2$ of eq. (8). Then, by equating the coefficients of $y^{i}(0 \leq i \leq$ 3 ) on both sides of eq. (9), we have

$$
\begin{aligned}
y^{3}: A_{2}^{\prime}(z)= & C(z) A_{2}(z), \\
y^{2}: A_{1}^{\prime}(z)= & C(z) A_{1}(z)+B(z) A_{2}(z), \\
y^{1}: A_{0}^{\prime}(z)= & C(z) A_{0}(z)+B(z) A_{1}(z) \\
& -2\left[\frac{\gamma+\alpha^{2}+\beta^{2}}{k^{2}\left(1+l^{2}\right)} z-\frac{\mu}{k^{2}\left(1+l^{2}\right)} z^{3}\right] A_{2}(z),
\end{aligned}
$$




\section{Ismail Aslan}

$$
y^{0}: B(z) A_{0}(z)-\left[\frac{\gamma+\alpha^{2}+\beta^{2}}{k^{2}\left(1+l^{2}\right)} z-\frac{\mu}{k^{2}\left(1+l^{2}\right)} z^{3}\right] A_{1}(z)=0 .
$$

As $A_{2}(z)$ and $C(z)$ are polynomials, from eq. (43) we deduce that $C(z)=0$ and $A_{2}(z)$ must be a constant. For simplicity, we can take $A_{2}(z)=1$. Balancing the degrees of $A_{2}(z), A_{1}(z)$ and $B(z)$, we conclude that $\operatorname{deg} B(z)=1$ only. Suppose that $B(z)=b_{1} z+b_{0}\left(b_{1} \neq 0\right)$. Then, we get

$$
\begin{aligned}
A_{1}(z)= & \frac{b_{1}}{2} z^{2}+b_{0} z+e \\
A_{0}(z)= & \frac{1}{4}\left(\frac{1}{2} b_{1}^{2}+\frac{2 \mu}{k^{2}\left(1+l^{2}\right)}\right) z^{4} \\
& +\frac{1}{2} b_{1} b_{0} z^{3}+\frac{1}{2}\left(e b_{1}+b_{0}^{2}-\frac{2\left(\gamma+\alpha^{2}+\beta^{2}\right)}{k^{2}\left(1+l^{2}\right)}\right) z^{2}+b_{0} e z+f,
\end{aligned}
$$

where $e$ and $f$ are integration constants. Substituting $A_{0}(z), A_{1}(z)$ and $B(z)$ into eq. (46) and setting the coefficients of $z^{i}(0 \leq i \leq 5)$ to be zero, we derive a system of nonlinear algebraic equations for $b_{0}, b_{1}, e, f, \mu, k, l, \alpha, \beta$ and $\gamma$. Solving the resultant system simultaneously, we get the solution set

$$
\begin{aligned}
& f=-\frac{\left(\alpha^{2}+\beta^{2}+\gamma\right)^{2}}{2 k^{2}\left(1+l^{2}\right) \mu}, \quad e=\mp \frac{\sqrt{2}\left(\alpha^{2}+\beta^{2}+\gamma\right)}{k \sqrt{1+l^{2}} \sqrt{-\mu}}, \\
& b_{0}=0, \quad b_{1}=\mp \frac{2 \sqrt{-2 \mu}}{k \sqrt{1+l^{2}}},
\end{aligned}
$$

where $k, l, \alpha, \beta$ and $\gamma$ remain arbitrary. Using the condition (49) in (8), we get

$$
y= \pm \frac{\alpha^{2}+\beta^{2}+\gamma-\mu z^{2}}{k \sqrt{1+l^{2}} \sqrt{-2 \mu}} .
$$

Combining eq. (42) with eq. (50) and changing to the original variables, we obtain an exact solution to eq. (39) as

$$
\begin{aligned}
u^{ \pm}(x, y, t)= & \pm \frac{\sqrt{\alpha^{2}+\beta^{2}+\gamma}}{\sqrt{-\mu}} \tan \left(\frac{\sqrt{\alpha^{2}+\beta^{2}+\gamma}}{k \sqrt{2\left(1+l^{2}\right)}}\left(k(x+l y+\lambda t)+\xi_{0}\right)\right) \\
& \times \exp (i(\alpha x+\beta y+\gamma t)),
\end{aligned}
$$

where $\mu<0$ and $\lambda=-2(\alpha+\beta l)$, while $k, l, \alpha, \beta, \gamma$ and $\xi_{0}$ remain arbitrary.

Remark 2. We note that our results are based on the assumptions $m=1$ and 2 in eq. (8). The discussion becomes more complicated for $m=3$ and 4 because the hyper-elliptic integrals, the irregular singular point theory, and the elliptic integrals of the second kind are involved. Also, we do not need to consider the case $m \geq 5$ because an algebraic equation with its degree greater than or equal to 5 is generally not solvable.

Remark 3. In the theory of nonlinear differential equations, searching for the first integrals of the nonlinear ordinary differential equations is one of the most important problem 


\section{Travelling wave solutions to nonlinear physical models}

because they permit us to get the general solution of a nonlinear differential equation in the form of quadratures. We observe that the first integral method, for discovering first integrals, can be applied to NPDEs which can be converted to a second-order ODE through the travelling wave transformation:

$$
\begin{aligned}
& u^{\prime \prime}(\xi)-\alpha P\left(u, u^{\prime}\right)-R(u)=0, \\
& u^{\prime \prime}(\xi)-Q\left(u, u^{\prime}\right) u^{\prime}(\xi)-R(u)=0,
\end{aligned}
$$

where $\alpha$ is real, $R(u)$ is a polynomial with real coefficients, $P(w, z)$ and $Q(w, z)$ are polynomials in $w$ and $z$.

\section{Conclusion}

As the investigation of the travelling wave solutions for NPDEs plays an important role in the study of nonlinear physical phenomena, it is worth trying new methods to tackle these types of equations. In this study, we focussed on the so-called first integral method for NPDEs. We analysed three equations for travelling wave solutions by using this method for the first time. It is observed that the method provides first integrals (in polynomial form) for planar systems in an efficient manner. But, there is still an open question which needs to be clarified: Can the procedure presented here be applied to NPDEs for multiwave solutions?

\section{Acknowledgement}

The author would like to thank an anonymous referee for the useful and valuable suggestions.

\section{References}

[1] R M Miura, Backlund transformation (Springer-Verlag, New York, 1973)

[2] J Weiss, M Tabor and G Carnevale, J. Math. Phys. 24, 522 (1983)

[3] M J Ablowitz and H Segur, Solitons and inverse scattering transform (SIAM, Philadelphia, 1981)

[4] G W Bluman and S Kumei, Symmetries and differential equations (Springer-Verlag, New York, 1989)

[5] R Hirota, The direct method in soliton theory (Cambridge University Press, Cambridge, 2004)

[6] C Yan, Phys. Lett. A224, 77 (1996)

[7] W Malfliet and W Hereman, Phys. Scr. 54, 563 (1996)

[8] G T Liu and T Y Fan, Phys. Lett. A345, 161 (2005)

[9] M Wang, X Li and J Zhang, Phys. Lett. A372, 417 (2008)

[10] J H He and X H Wu, Chaos, Solitons and Fractals 30, 700 (2006)

[11] Z Feng and X Wang, Phys. Scr. 64, 7 (2001)

[12] Z Feng, Phys. Lett. A293, 57 (2002)

[13] Z S Feng, J. Phys. A: Math. Gen. 35, 343 (2002)

[14] Z Feng and R Knobel, J. Math. Anal. Appl. 328, 1435 (2007)

[15] X Deng, Appl. Math. Comput. 204, 733 (2008) 


\section{Ismail Aslan}

[16] Naranmandula and K X Wang, Chin. Phys. 13, 139 (2004)

[17] H Li and Y Guo, Appl. Math. Comput. 180, 524 (2006)

[18] K R Raslan, Nonlin. Dyn. 53, 281 (2008)

[19] K Yun-Quan and Y Jun, Commun. Theor. Phys. 43, 597 (2005)

[20] J Lu, G Yu-Cui and X Shu-Jiang, Chin. Phys. 16, 2514 (2007)

[21] S Abbasbandy and A Shirzadi, Commun. Nonlinear Sci. Numer. Simulat. 15, 1759 (2010)

[22] T R Ding and C Z Li, Ordinary differential equations (Peking University Press, Peking, 1996)

[23] Z Feng, Electron J. Linear Algebra 8, 14 (2001)

[24] N Bourbaki, Commutative algebra (Addison-Wesley, Paris, 1972)

[25] R K Dodd et al, Soliton and nonlinear waves (Academic Press, London, 1982)

[26] Z Y Yan, F D Xie and H Q Zhang, Commun. Theor. Phys. 36, 1 (2001)

[27] G B Whitham, Proc. R. Soc. London Ser. A299, 6 (1967)

[28] R Ivanov, J. Nonlinear Math. Phys. 1294, 462 (2005)

[29] B Fornberg and G B Whitham, Philos. Trans. R. Soc. London Ser. A289, 373 (1978)

[30] J Zhou and L Tian, J. Math. Anal. Appl. 346, 255 (2008)

[31] J Zhou and L Tian, Nonlinear Anal. Real World Appl. 11, 356 (2010) 
Copyright of Pramana: Journal of Physics is the property of Indian Academy of Sciences and its content may not be copied or emailed to multiple sites or posted to a listserv without the copyright holder's express written permission. However, users may print, download, or email articles for individual use. 